\title{
Complexity of care and strategies of self- management in patients with colorectal cancer
}

\author{
This article was published in the following Dove Press journal: \\ Patient Preference and Adherence \\ 10 April 2017 \\ Number of times this article has been viewed
}

\section{Dominik Ose ${ }^{1,2}$ \\ Eva CWinkler ${ }^{3}$ \\ Sarah Berger' \\ Ines Baudendistel' \\ Martina Kamradt' \\ Felicitas Eckrich' \\ Joachim Szecsenyi ${ }^{1}$ \\ 'Department of General Practice and Health Services Research, University Hospital Heidelberg, Heidelberg, Germany; ${ }^{2}$ Department of Population Health, Health System Innovation and Research, University of Utah, Salt Lake City, UT, USA; ${ }^{3}$ Program for Ethics and Patient-oriented Care in Oncology, National Centre for Tumour Diseases, University Hospital Heidelberg, Heidelberg, Germany}

Correspondence: Dominik Ose University of Utah, Department of Population Health Sciences, Health System Innovation and Research, Williams Building, 295 Chipeta Way,

Salt Lake City, UT 84108, USA

$\mathrm{Tel}+\mathrm{I} 80$ I 5872263

Fax + I 80I 58I 3623

Email dominik.ose@hsc.utah.edu
Purpose: Given the inherent complexity of cancer care, in which personal, social, and clinical aspects accumulate and interact over time, self-management support need to become more comprehensive. This study has the following two aims: 1) to analyze and describe the complexity of individual patient situations and 2) to analyze and describe already established self-management strategies of patients to handle this complexity.

Methods: A qualitative study was conducted. Ten focus groups were performed collecting perspectives of the following three user groups: patients with colorectal cancer $(n=12)$ and representatives from support groups $(n=2)$, physicians $(n=17)$, and other health care professionals (HCPs; $n=16$ ). Data were analyzed using qualitative content analysis.

Results: The results showed that cancer patients are struggling with the complexity of their individual situations characterized by the 1) "complexity of disease", 2) "complexity of care", and 3) "complexity of treatment-related data". To deal with these multifaceted situations, patients have established several individual strategies. These strategies are "proactive demanding" (eg, to get support and guidance or a meaningful dialog with the doctor), "proactive behavior" (eg, preparation of visits), and "proactive data management" (eg, in terms of merging treatmentrelated data and to disseminate these to their health care providers).

Conclusion: Patients with colorectal cancer have to handle a high complexity of individual situations within treatment and care of their disease. Private and social challenges have a culminating effect. This complexity increases as patients experience a longer duration of treatment and follow-up as patients have to handle a significantly higher amount of data over time. Self-management support should focus more on the individual complexity in a patient's life. This includes assisting patients with strategies that have already been established by themselves (like preparation of visits).

Keywords: self-management, health care utilization, colorectal cancer, chronic care, health services research, complexity

\section{Introduction}

With the steady progress in cancer treatment and survival, ${ }^{1,2}$ as well as an aging population, cancer prevalence is increasing. Some cancers (eg, colorectal cancer) have shown improvements in 5-year survival, while other high-prevalence cancers (eg, lung cancer) have not. Cancer can be a high symptom burden disease with rapid decline, as well as a chronic disease. ${ }^{3,4}$

However, chronic care of patients with cancer is a complex process involving multiple and cyclic diagnostics and treatments. The majority of patients and their families have to deal with several dimensions of burden (eg, symptoms, disabilities, and uncertainty) and experience different care settings with input from various medical specialists (eg, oncologist, surgeon, and gastroenterologist) as well as care and support 
from interprofessional teams over time, eg, psychologist, therapist, and nurse..$^{5-7}$

Similar to other chronic diseases, this includes a substantial part of self-management in terms of interacting with health care providers, monitoring health status, making health care-related decisions, and managing the impact of the illness on physical functioning, well-being, and social relationships. ${ }^{8}$ Overall, self-management includes all tasks "that individuals undertake to deal with the medical, role, and emotional management of their health condition(s)". 9

Concepts of self-management have been primarily implemented in other chronic illness populations such as patients with diabetes. Compared with existing evidence for other chronic illness populations, the number of studies about self-management among cancer patients is limited. ${ }^{10-13}$ However, in recent years some cancer-specific concepts for self-management support have also been developed. ${ }^{14-16}$ In this context, self-management support is described as those actions that can be performed by health services to support people living with long-term conditions to improve or maintain their health and well-being. ${ }^{17}$

Supporting patients to be active participants in their health journey from diagnosis to long-term survival can offer patients an increased level of autonomy in their health and may enhance overall quality of life (QoL). ${ }^{18-21}$ Current studies with colorectal cancer patients have shown that selfmanagement support can enhance self-efficacy ${ }^{22}$ and can help with ostomies adapt to their stoma ${ }^{23}$ and to guide future self-care behavior. ${ }^{24}$ On the other hand, lower self-efficacy was significantly associated with poorer trajectories for QoL, health status, and well-being after adjusting for disease characteristics, stoma, anxiety, and social support. ${ }^{25}$

However, current interventions to support self-management are often more focused on certain aspects of disease (eg, dealing with symptoms) and less on health service utilization as well as private or social challenges. ${ }^{18}$ Given the inherent complexity of cancer care, self-management support need to become more comprehensive. Complexity in this context can be characterized as a dynamic state in which the personal, social, and clinical aspects of the patient's experience work as complicating factors that interact with each other and accumulate over time. ${ }^{26}$ More evidence is needed on how cancer patients manage to live with persistent problems arising from treatment and how they can best be supported in self-management of encountered problems. ${ }^{27-31}$

This study has the following two aims: 1) to analyze and describe the complexity of individual patient situations and 2) to analyze and describe already established self-management strategies of patients in handling this complexity. The results of this study can contribute to the further development of concepts for self-management support, which is centered on the patient perspective and needs.

\section{Methods}

A project called "Information technology for patient-centered healthcare" (INFOPAT) funded by the German Federal Ministry of Education and Research (2012-2016) has been initiated in the Rhine-Neckar region in Germany. ${ }^{32,33}$ INFOPAT aims to improve care across different health care settings, especially for chronically ill patients. In the first phase of the INFOPAT project, experiences of patients and health care professionals (HCPs) in the field of colorectal cancer care are being investigated. The research project was approved by the ethics committee of the University Hospital Heidelberg (S-497-2012). All participants gave their written informed consent. The participants' anonymity and confidentiality have been preserved throughout the reported study. All procedures involving human participants were in accordance with the ethical standards of the institutional and/or national research committee and with the 1964 Declaration of Helsinki and its later amendments or comparable ethical standards.

\section{Study design}

Based on the explorative character of the research topic, the research team decided to use a qualitative approach and performed focus groups to collect appropriate initial data. The decision to collect data through focus groups was based on the explorative character of the research topic. A focus group is a group interview with participants who are involved in the research field of interest. ${ }^{34}$ Because the topic of this study is fairly new, the group processes should help our participants to explore and clarify their views and attitudes.

\section{Study sample and recruitment}

As described previously, ${ }^{35}$ a wide range of different views on colorectal cancer care had to be explored within the INFOPAT project. Eligible participants were patients diagnosed with colorectal cancer (Eastern Cooperative Oncology Group [ECOG] 0-1; at least 18 years old and not suffering from severe acute psychiatric disorders or moderate to severe dementia), representatives from patient support groups, and physicians and other HCPs working in the Rhine-Neckar region, Germany.

Patients were recruited through the National Center for Tumor Diseases (NCT) in Heidelberg, Germany, where they 
also received their cancer treatment. Further patients were approached through an umbrella organization for patient support groups in Heidelberg. Recruited physicians were involved in colorectal cancer care at the NCT (oncology specialists) and in ambulatory settings (GPs and other registered medical specialists). Other participating HCPs (nurses, stoma therapists, social workers, a physiotherapist, and a nutritionist) worked either at the NCT or the University Hospital Heidelberg and were involved in colorectal cancer care. Additionally, health care assistants ${ }^{36}$ who were working in primary care practices were included.

\section{Data collection}

The research team collaborative developed a semistructured interview guide with open-ended questions, which was intended to obtain and explore the participants' experiences in colorectal cancer care. Themes and questions of the interview guide were based on theoretical considerations, expert discussions, and an extensive literature review. This approach was in accordance with the principle of qualitative research to be theory driven. The principle of openness was taken into account by asking questions that allowed participants a broad discussion. ${ }^{34,37,38}$

This interview guide was pretested and following minor fine tuning (eg, used terms) was later used by experienced researchers (IB, DO, ECW, and FE) to facilitate the focus groups. The focus groups were held at the University Hospital Heidelberg, and recruitment for new participants continued until thematic saturation was reached in that no new aspects emerged. All focus group discussions were audio- and videotaped and later transcribed verbatim. Videotapes were used to assist with the transcription of group data. Additionally, sociodemographic data were collected anonymously using a study short questionnaire developed for this study phase.

\section{Data analysis}

This qualitative content analysis ${ }^{39}$ is based on the following question out of our focus groups: "What are the challenges in cross-sectoral treatment and care in patients with colorectal cancer?" For analysis, data were taken from the transcribed texts, coded, and analyzed in a stepwise process. In a first step, transcriptions were analyzed independently by three researchers (IB, DO, and MK) to identify relevant key issues using a preliminary category system (search grid). This was based on theoretical considerations of patient-centered care and a literature review regarding personal health records. In addition, the category system was adapted during the process of analysis if the data showed additional and new information that did not fit into the previous category system.

In a second step, the key findings were discussed with the research team. Later, all key issues were labeled as codes and organized into main categories and subcategories. Each code was clearly defined and linked to text examples from the transcriptions. Finally, all categories were discussed within the research team and modified until consensus on the final category system was reached. Labeling categories were performed using ATLAS.ti (version 7.0.80).

\section{Results}

\section{Presentation of results}

For reader friendliness, the tables highlight the key findings as main categories as well as the corresponding subcategories and aspects of each subcategory. Tables present the categories and enable differentiation between the perspectives of different participant groups with respect to mentioned aspects. In addition, selected quotations are mentioned in the text. An overview of all relevant quotations can be found in Tables S1 and S2.

\section{Focus groups and participants}

Ten focus groups with a total of 47 participants were conducted (March until October 2013). Three focus groups were with patients $(n=12)$ and representatives from patient support groups $(n=2)$, four focus were groups with physicians $(n=17)$, and three focus groups were with other HCPs $(n=16)$ (Table 1). The characteristics of the focus group participants are presented in Table 2.

\section{Complexity of individual situations}

Overall, our focus group participants stated that cancer patients are struggling to deal with the complexity of this disease. This included not only the complexity of medical treatment but also psychological aspects and challenges in social life. In our focus groups, aspects of the complexity of individual situations were discussed in the context of different topics: complexity of disease, complexity of care, and complexity of treatment data (Table 3). These are important for the understanding of self-management strategies and will be described in detail subsequently.

\section{Complexity of disease}

In terms of complexity of disease, one issue in our focus groups was the "side effects of chemotherapy". The point that patients have to live with side effects (sometimes 
Table I Demographics and participants characteristics

\begin{tabular}{|c|c|c|c|c|}
\hline Characteristics & Patients & Patient representatives $^{a}$ & Nonphysician HCPs & Physicians \\
\hline $\mathrm{n}$ & 12 & 2 & 16 & 17 \\
\hline Sex (male) & $83.3 \%(n=10)$ & $50.0 \%(n=1)$ & $18.8 \%(n=3)$ & $58.8 \%(n=10)$ \\
\hline Age (years) ${ }^{b}$ & $61.5(58.0 ; 67.2)$ & $(44 ; 62)^{c}$ & $38.0(28.5 ; 50.0)$ & $43.0(35 ; 56.5)$ \\
\hline Education $\geq 12$ years & $50.0 \%(n=6)$ & $100.0 \%(n=2)$ & $43.7 \%(n=7)$ & - \\
\hline Professional experience (years) ${ }^{b}$ & - & $(10 ; 38)^{c}$ & $20(5.0 ; 26.0)$ & $15(5.0 ; 26.5)$ \\
\hline \multicolumn{5}{|l|}{ Health care setting } \\
\hline $\mathrm{NCT}^{\mathrm{d}}$ & - & - & $75 \%(n=12)$ & $29.4 \%(n=5)$ \\
\hline Outpatient care ${ }^{e}$ & - & - & $25 \%(n=4)$ & $70.6 \%(n=12)$ \\
\hline
\end{tabular}

Notes: a Patient representatives $=$ staff from patient support groups. ${ }^{\mathrm{b} M d}(\mathrm{IQR}) .{ }^{\mathrm{c}}$ Minimum; maximum. ${ }^{\mathrm{d} N C T}$ : oncological specialists. ${ }^{\mathrm{e} G e n e r a l}$ practitioners, registered specialists. Abbreviations: HCPs, health care professionals; NCT, National Center for Tumor Diseases; Md, median; IQR, interquartile range.

severe) of chemotherapy was pointed out by all participants. Side effects varied from nausea, constipation, headache, hair loss, and inflammation to severe depression and many others. Dealing with the different side effects constituted a huge challenge for patients and was an aspect that made the disease more complex.

I am really satisfied with the therapy except that I have just nausea and constipation and diarrhea and headache, and inflammation in the mouth and so on, and so on, so that comes regularly. [FG1-P2]

Within the focus groups, it was also recognizable that as a "consequence of the cancer treatment", patients also had a range of practical problems, for example, difficulties in managing an ostomy or impaired wound healing. Moreover, with increasing age and survival time, patients suffered not only from cancer but also from additional diseases (eg, type 2 diabetes or hypertension). Associated with this "co- and multimorbidity" was an increasing number of treatments and interventions that were not always compatible with each other (eg, medication interactions). Both "consequence of treatment" and existing co- and multimorbidities increased the complexity dramatically and further increased as patients had to handle "anxiety and uncertainty" about the development of the disease and its possible recurrence.

\section{Complexity of care}

During the discussions, several focus group participants highlighted the importance of "interprofessional and intersectoral cooperation" in cancer care. Treatment of tumor patients requires close cooperation of providers in both outpatient and inpatient services (eg, GP and hospital) as well as a close cooperation between all health professionals involved in the patient's care (eg, doctors, nutrition, and therapists). Not only for patients but also for the involved HCPs, cancer care is very complex in service delivery and organization.

I was passed around in the clinic [...] also previously at the preliminary investigation I was at the [...] urologist because $[\ldots]$ the family doctor had a suspicion that I might have kidney stones $[\ldots]$ the urologist sent me to the surgeon and the surgeon sent me to the gynecologist and there I got caught. [FG3-P2]

Moreover, with improved treatment options and an increasing survival time, "long-term care" in combination with "importance of homecare" and the involvement of caregivers are becoming central patient needs. For patients, the significance of "private and social challenges" is closely linked to these needs. As revealed by our focus group participants, patients have to deal with complex individual and social situations due to the cancer diagnosis, for example, in terms of employment, financial security, and consequences

Table 2 Compositions of conducted focus groups $(n=10)$

\begin{tabular}{llll}
\hline User group & Number & Description \\
\cline { 2 - 4 } & Focus groups (n) & Participants (total N) & \\
\hline Patients & 3 & 14 & $\begin{array}{l}\text { Patients with colorectal cancer, representatives } \\
\text { from patient support groups }\end{array}$ \\
Physicians & 4 & 17 & Oncological specialists, GPs, registered specialists \\
Nonphysician HCPs & 3 & 16 & Nurses, social workers, physiotherapists, \\
Total & 10 & 47 & nutritionists, health care assistants \\
\hline
\end{tabular}

Abbreviation: HCPs, health care professionals. 
Table 3 Complexity of individual situations

\begin{tabular}{ll}
\hline Category & Subcategory \\
\hline Complexity of disease & Side effects of chemotherapy \\
& Anxiety and uncertainty \\
Consequences of cancer treatment & b, a, c \\
b, a & Co- and multimorbidity \\
Complexity of care & Interprofessional and intersectoral cooperation \\
& Importance of long-term and home care \\
Complexity of treatment data & Private and social challenges \\
& Increasing amount of data \\
b, c, a & Various data sources and numerous data types \\
& Difficult traceability and assignment \\
\hline
\end{tabular}

Notes: a, patients; b, physicians; c, nonphysician HCPs.

Abbreviation: HCPs, health care professionals.

in terms of changes in relationships with their families. Quite often family members have an important role to play in the support of cancer patients who are dealing with their complex personal situation.

\section{Complexity of treatment-related data}

In contrast to other cancer diseases (eg, lung cancer), patients with colorectal cancer tend to have longer survival times. With increasing duration of treatment and follow-up, these patients have to deal with an "increasing amount of data" related to their care. Because of the complexity of this disease (eg, in terms of co- and multimorbidity) and the complexity of care (eg, in terms of interprofessional and intersectoral cooperation), data are produced in multiple settings by many different providers. Within the focus groups, it became apparent that with an increasing duration of disease and an increasing amount of data, it becomes more difficult for patients to keep track of treatment relevant data ("difficult traceability and assignment"). Additionally, patients had to handle a "variety of data sources and numerous data types" (eg, reports, medication plans, and X-rays) whose individual significance for the further course of treatment was difficult to determine.

If the patient has collected results over the past 2 years, it is difficult to decide what is relevant or what not. [FG4-Doc3]

\section{Strategies of self-management}

To face the challenges described earlier, patients have developed a variety of strategies. The foundation of these strategies is that they all require a proactive patient role. Overall, the strategies are related to the handling of the complexity of disease, the complexity of care, and the complexity of treatment data. The strategies that were mentioned in our focus groups are presented in the following section (Table 4).

\section{Proactive demanding}

In cancer treatment and organization of care, patients often feel alone and overwhelmed by the described complexity. In such situations, patients in our focus groups experienced that dealing with challenges was easier if they proactively sought to have their needs met. This applied also to the utilization of health care services. The organization of interprofessional and intersectoral care was quite often a huge challenge. Consistently, patients reported in certain contexts that they only got additional "support and guidance" when requested.

And then I said "please could you tell me where to go now" and then they told me the next steps and made a phone call and took action. [FG1-P4]

Another relevant topic in our discussions was the communication between patient and physicians. Besides the actual therapy, topics such as side effects of chemotherapy, consequences of cancer treatment, and the dealing with co- and multimorbidity were very relevant for patients. However, repeatedly patients pointed out that it was not easy to track down the appropriate health professional who had the time (from patient perspective) to talk with them ("meaningful talk with the doctor"). Although patients had a primary

Table 4 Strategies of self-management

\begin{tabular}{lll}
\hline Category & Subcategory & Mentioned by \\
\hline Proactive & Support and guidance & c, a, b \\
demanding & Meaningful talk with the doctor & a, c \\
& Therapy and treatment options & a, b \\
Proactive behavior & Organization of care & a, c \\
& Preparation of visits & a, b \\
Proactive data & Searching for information & b, a \\
management & Merging of treatment data & a, c \\
& Dissemination of information & a, b, c \\
& to providers & \\
\hline
\end{tabular}

Notes: a, patients; b, physicians; c, nonphysician HCPs.

Abbreviation: $\mathrm{HCPs}$, health care professionals. 
doctor, they often did not agree with the manner in which conversations took place. In particular, many patients are dissatisfied with the talks about the diagnosis or prognosis (eg, because too little focused on individual perspective and needs). Often patients have to ask proactively for a meaningful talk with the doctor.

On a surgical ward, I actively sought a conversation and then I complained. Then someone had time to talk with me. But it is a shame when one has to resort to such steps.

[FG3-P6]

In addition, in terms of "therapy and treatment options", patients had the feeling that their specific needs were not always adequately considered (eg, in terms of information). In particular, for patients with a long treatment history, in combination with different care settings and providers, this constituted a significant challenge. However, patients in our focus groups did report that they explicitly confronted their doctors about their treatment results and actively asked for a medical opinion on how to proceed. This included not only next steps but also concrete options for long-term therapy and treatment.

I took my medical records to the clinic and said "so can we take a look at this together and can we discuss what it means". [FG3-P4]

\section{Proactive behavior}

Even if active requests for aspects of care was successful (eg, guidance), a lot of responsibility remained on patients for organizing and managing their own care. This includes not only the coordination of care and the arrangement of appointments but also active "demands". Some patients believed that without their proactive behavior in the "organization of their care", a number of things would not have worked out.

I always took action. Whether it would have worked without this I do not know. But I've always pushed. [FG1-P4].

The importance of "preparation of visits" was highlighted within the focus groups. In this context, patients repeatedly pointed out that preparation for communication with physicians and other relevant HCPs had a special meaning in their cancer care. Patients often had the impression that physicians had limited time and did not always know all the details of their treatment history. In this situation, for patients it proved important to prepare relevant topics and a discussion plan beforehand. This means that patients wrote down important questions and defined goals before talking with the doctor.

My experience is that it is very helpful if you are well informed going to the doctor [...] all doctors - I think that's just a certain human trait - respond differently to patients who coming informed. [FG2-P1]

Regardless of this situation, physicians and other HCPs are key persons for patients in their search for health- and treatment-related information. Additionally, in recent years other sources of information, such as the Internet, have become more important. These additional sources were used by most patients in our focus groups. The "search for information" was a relevant strategy of self-management because patients had experienced that information from physicians and other HCPs did not always turn out to be correct or that it was contradictory. The search for information on the Internet as well as in books and in brochures was for many patients a crucial aspect of gathering health care information.

I then asked what I can actually eat and then they said "everything". Then I had to learn [that this is not true].

However, there are excellent booklets on nutrition for cancer patients. [FG2-P2]

\section{Proactive data management}

Another major challenge and at the same time relevant strategy of self-management is the "merging of treatment data". Patients with a long treatment history - often combined with different care settings - have to handle a significant amount of data over the course of their illness. Considering the complexity of the treatment, disease- and care-related data are collected from many different service providers. Our participants had the impression that they often had responsibility to bring all their data together. It became apparent with our focus groups that much time and commitment were needed for patients to merge all of their treatment data. The patients in our focus groups often knew exactly how they receive their treatment data from different health care providers (eg, format of data, contact person, etc.).

I have all the reports. Because I stuck to my guns and said

"hey I still lack such and such and that is still missing" and

so on. So it worked well on request. [FG3-P2]

The described attentiveness in terms of complete treatment data was also necessary for the "dissemination of information to providers". From the viewpoint of our focus group 
participants, the responsibility for the transfer of treatment data between providers often had to be taken over by patients. Moreover, our participants experienced that they were sent away, if the relevant documents were not complete.

And I'll tell it to anyone [...] they should take and distribute their medical results $[\ldots]$ if the patient does not have an extra copy for the doctors, then he may will be sent back home. [FG3-P1]

Last but not least, in terms of proactive data management, many patients reported their efforts to archive their treatment data sustainably ("archiving of documents"). For patients with a treatment history of 5 years and more, the volume of treatment-related data exceeded rapidly the capacity of several case binders. Therefore, some of the patients in our focus groups made electronic scans of their documents for a digital archive.

\section{Discussion}

The first aim of this study was to analyze and describe the complexity of individual patient situations. The results demonstrated that patients with colorectal cancer are challenged by this complexity. In terms of the "complexity of disease", our results indicated that dealing with different side effects (eg, depression), practical problems (eg, managing an ostomy), and existing co- and multimorbidities culminate and increase the complexity of disease dramatically. This process has also been recognized in terms of the "complexity of care". This culminated as the following factors came into play: fragmentation across intersectoral care (eg, between GP and hospital) and interprofessional teams (eg, doctors, homecare, and nutrition therapist), as well as private and social challenges. It also increased as patients experienced a longer duration of treatment and follow-up. Those patients had to handle a significantly higher amount of data from various data sources and numerous data types ("complexity of treatment-related data"). While the importance the complexity of treatment-related data represents an novel finding, the others are in line with previous literature. ${ }^{26,40}$

The second aim of this study was to analyze and describe established self-management strategies of patients to handle this complexity. In terms of "proactive demanding", patients established strategies to get support and guidance, a meaningful talk with the doctor, and therapy and treatment options. Moreover, pushing the organization of care, preparation of visits with physicians and other HCPs, as well as searching for information were relevant self-management strategies in the context of "proactive behavior". Additionally, in terms of "proactive data management" merging treatment-related data, the dissemination of information to providers and archiving treatment data were also important strategies for many patients.

Previous research has rarely addressed aspects of individual self-management strategies in patients with cancer. ${ }^{17}$ One approach that describes strategies of cancer patients in the management of problems associated with their conditions was developed by Foster et al. ${ }^{41}$ This model comprises two main components: sources of self-management support (eg, health care workers, family, and accessing information) and self-management strategies. In terms of self-management strategies, the model distinguishes between strategies for psychological problems (eg, managing emotions), strategies for social problems (eg, managing work), and strategies for physical problems. ${ }^{28,41}$

Overall, this approach is strongly orientated toward a psychoeducational understanding of self-management in the tradition of the concept of Lorig and Holman. ${ }^{42}$ In this context, self-management support should enable patients to perform primarily the following three sets of tasks: 1) medical management of their illness in terms of taking medication and being adherent to treatment regime, 2) carrying out normal roles and activities, and 3) managing the emotional impact of illness. ${ }^{42}$

Strategies to support the utilization of health services or to support data management are not part of both concepts. However, the concept of Lorig and Holman arose at a time, in which the patient-doctor relationship was of primary focus. Aspects of intersectoral and interprofessional chronic care have only begin in the last decade to receive attention. ${ }^{9}$

In the broader context of chronic diseases, previous research about strategies of self-management exists. For example, Audulv ${ }^{43}$ distinguishes between "consistent" (eg, long-term medication), "on demand" (eg, seeking information and navigating health care), "episodic" (eg, exercise), and "transitional" (eg, managing household activities) selfmanagement strategies. Similarities to our findings exist in terms of the importance of self-management for the utilization of health services and the consideration of household activities. The main difference to our results is the consideration of self-management patterns over time. ${ }^{43}$

The results of Schulman-Green et $\mathrm{al}^{44}$ are stronger orientated on the process of self-management as our findings Based on a structured literature review, they identified the following three categories of self-management processes: focusing on illness needs (eg, taking ownership of health needs), activating resources (eg, navigating the health care 
system), and living with a chronic illness (eg, integrating illness into daily life). ${ }^{44}$ In terms of specific diseases, Pound et $\mathrm{al}^{45}$ describe "mobilizing informal social support", "beginning a process of relearning", or "creating new ways of doing things" as important strategies of self-management for stroke. Important self-managing strategies for people living with antiretroviral therapy in Uganda are "obtaining medications", "adjustment of new roles" or "managing disclosure and family". 46

However, adequate self-management support for persons with complex chronic diseases may differ from that needed for persons with single diseases in process and content. To date, only a few studies considered aspects of selfmanagement for patients with high complexity of treatment, care, and social demands, ${ }^{47-49}$ this is also true for patients with colorectal cancer. ${ }^{50-52}$ The unique feature of our analysis is to bringing both aspects together: complexity of care and strategies to handle this complexity. Additionally, our results highlight the increased importance of personal health information and the need for advanced concepts for selfmanagement support. The increasing importance of health information exchange and the role of patients in this context ${ }^{35}$ are critical and should be given much more attention.

\section{Implications for practice}

Today, a broad range of different concepts for selfmanagement support exists. Besides traditional approaches, which aim to support the coping process (eg, in terms of psychological, social, and physical problems), more recent approaches are stronger oriented toward the utilization of health care. The importance of moving in this direction is reflected with our results. Future concepts should strongly incorporate these findings.

In terms of "complexity", the concept of Shippee et $\mathrm{al}^{26}$ describes a mechanism whereby complicating factors impact care trajectories and outcomes, ie, the balance between patient demands and patient capacity to address demands. ${ }^{26}$ Taking this approach as a starting point, concepts of self-management can take individual complexity and individual resources more into account. This seems to be highly relevant.

Patients often strive for normality, whereas their health situation may demand daily assessment of biophysical needs, implementation of appropriate management strategies, and evaluation of treatment effectiveness. Without knowing the rationale of self-management, patients often fail to implement these strategies. ${ }^{53}$ On the other hand, our analysis has revealed that patients have established already own self-management strategies. Learning more about these and supporting patients in what they already do are important implications of this study.

However, this requires to address more specific the shortcomings of our health care systems (eg, lack of continuity of care and lack of interprofessional communication). Then we can more effectively support patients to deal with these situations. Many of the highlighted strategies are an attempt to compensate for existing challenges of health care utilization.

\section{Strength and weaknesses}

This analysis has a strong focus on the delivery and utilization of health care. This is a strength, because the consideration of self-management support is often predominantly focused on the adherence and a psychoeducational understanding of self-management. In this way, our results can contribute a broader perception of self-management support. However, it is also a weakness because it could give the wrong impression that disease-related aspect (eg, the handling of side effects) or psychosocial aspects (eg, managing the emotional impact of the disease) are less important for self-management support. This is not the case.

However, to collect a wide range of experiences, this study incorporated a mixed sample of participants who are involved in colorectal cancer care and considered cancer care at all stages of the disease. Because we understand colorectal cancer care as an interprofessional collaborative effort to deliver health services for patients, we included patients as well as physicians and other HCPs from different health care settings in our study sample. However, most patients willing to participate in the study were men, and the level of education was relatively high. Therefore, the findings may not be generalizable to the general colorectal cancer patient population. The number of participants (in particular of patients) was too low. It is possible that a higher number of participants would have led to different findings. Unfortunately, we do not have any information about tumor stage, medical treatment, or other covariables. These aspects may have an impact on our results. In terms of analysis, interrater reliability was not measured.

\section{Conclusion}

Patients with colorectal cancer have to handle a high complexity of individual situations within treatment and care of their disease. Private and social challenges have a culminating effect. This complexity increases as patients experience a longer duration of treatment and follow-up as patients have 
to handle a significantly higher amount of data over time. Interventions for self-management support should focus more on the individual complexity in a patient's life. This includes assisting patients with strategies that have already been established by themselves (like preparation of visits).

\section{Acknowledgment}

This study was funded by the German Federal Ministry of Education and Research (funding code 01KQ1003B).

\section{Disclosure}

The authors report no conflicts of interest in this work.

\section{References}

1. Hiripi E, Gondos A, Emrich K, et al. Survival from common and rare cancers in Germany in the early 21st century. Ann Oncol. 2012;23(2): 472-479.

2. Jansen L, Castro FA, Gondos A, et al. Recent cancer survival in Germany: an analysis of common and less common cancers. Int $J$ Cancer. 2015;136(11):2649-2658.

3. Wang H, Liddell CA, Coates MM, et al. Global, regional, and national levels of neonatal, infant, and under-5 mortality during 1990-2013: a systematic analysis for the Global Burden of Disease Study 2013. Lancet. 2014;384(9947):957-979.

4. Kassebaum NJ, Bertozzi-Villa A, Coggeshall MS, et al. Global, regional, and national levels and causes of maternal mortality during 1990-2013: a systematic analysis for the Global Burden of Disease Study 2013. Lancet. 2014;384(9947):980-1004.

5. Sussman J, Baldwin L-M. The interface of primary and oncology specialty care: from diagnosis through primary treatment. J Natl Cancer Inst Monogr. 2010;2010(40):18-24.

6. Hewitt ME, Ganz P. From Cancer Patient to Cancer Survivor. Lost in Transition. Washington, DC: National Academies Press; 2006.

7. Fleissig A, Jenkins V, Catt S, Fallowfield L. Multidisciplinary teams in cancer care: are they effective in the UK? Lancet Oncol. 2006;7(11): 935-943.

8. Clark NM, Becker MH, Janz NK, Lorig K, Rakowski W, Anderson L. Self-management of chronic disease by older adults a review and questions for research. J Aging Health. 1991;3(1):3-27.

9. McCorkle R, Ercolano E, Lazenby M, et al. Self-management: enabling and empowering patients living with cancer as a chronic illness. CA Cancer J Clin. 2011;61(1):50-62.

10. Gao WJ, Yuan CR. Self-management programme for cancer patients: a literature review. Int Nurs Rev. 2011;58(3):288-295.

11. Du S, Hu L, Dong J, et al. Self-management program for chronic low back pain: a systematic review and meta-analysis. Patient Educ Couns. 2017;100(1):37-49.

12. Hood M, Wilson R, Corsica J, Bradley L, Chirinos D, Vivo A. What do we know about mobile applications for diabetes self-management? A review of reviews. J Behav Med. 2016;39(6):981-994.

13. Stenberg U, Haaland-Overby M, Fredriksen K, Westermann KF, Kvisvik T. A scoping review of the literature on benefits and challenges of participating in patient education programs aimed at promoting self-management for people living with chronic illness. Patient Educ Couns. 2016;99(11):1759-1771.

14. Davies NJ, Batehup L. Self-Management Support for Cancer Survivors: Guidance for Developing Interventions: An Update of Evidence. London: National Cancer Survivorship Initiative; 2010.

15. Coffey L, Mooney O, Dunne S, et al. Cancer survivors' perspectives on adjustment-focused self-management interventions: a qualitative meta-synthesis. J Cancer Surviv. 2016;10(6):1012-1034.
16. Cockle-Hearne J, Faithfull S. Self-management for men surviving prostate cancer: a review of behavioural and psychosocial interventions to understand what strategies can work, for whom and in what circumstances. Psychooncology. 2010;19(9):909-922.

17. Fenlon D, Foster C. Self Management Support: A Review of the Evidence. London: National Cancer Survivorship Initiative; 2009.

18. Hammer MJ, Ercolano EA, Wright F, Dickson VV, Chyun D, Melkus GD. Self-management for adult patients with cancer: an integrative review. Cancer Nurs. 2015;38(2):26.

19. Ose D, Rochon J, Campbell SM, et al. Health-related quality of life and risk factor control: the importance of educational level in prevention of cardiovascular diseases. Eur J Public Health. 2014;24(4):679-684.

20. Phillips J. The need for an integrated approach to supporting patients who should self manage. SelfCare. 2012;3(2):33-41.

21. Jordan JE, Briggs AM, Brand CA, Osborne RH. Enhancing patient engagement in chronic disease self-management support initiatives in Australia: the need for an integrated approach. Med J Aust. 2008; 189(10 suppl):S9-S13.

22. Zhang M, Chan SW-C, You L, et al. The effectiveness of a self-efficacyenhancing intervention for Chinese patients with colorectal cancer: a randomized controlled trial with 6-month follow up. Int J Nurs Stud. 2014;51(8):1083-1092.

23. Krouse RS, Grant M, McCorkle R, et al. A chronic care ostomy selfmanagement program for cancer survivors. Psychooncology. 2016; 25(5):574-581.

24. Rosenberg CA, Flanagan C, Brockstein B, et al. Promotion of selfmanagement for post treatment cancer survivors: evaluation of a riskadapted visit. J Cancer Surviv. 2016;10(1):206-219.

25. Foster C, Haviland J, Winter J, et al. Pre-surgery depression and confidence to manage problems predict recovery trajectories of health and wellbeing in the first two years following colorectal cancer: results from the CREW cohort study. PLoS One. 2016;11(5):e0155434.

26. Shippee ND, Shah ND, May CR, Mair FS, Montori VM. Cumulative complexity: a functional, patient-centered model of patient complexity can improve research and practice. J Clin Epidemiol. 2012;65(10): 1041-1051.

27. Richards M, Corner J, Maher J. The National Cancer Survivorship Initiative: new and emerging evidence on the ongoing needs of cancer survivors. Br J Cancer. 2011;105(suppl 1):4.

28. Foster C, Fenlon D. Recovery and self-management support following primary cancer treatment. Br J Cancer. 2011;105(suppl 1):8.

29. Sparla A, Flach-Vorgang S, Villalobos M, et al. Individual difficulties and resources - a qualitative analysis in patients with advanced lung cancer and their relatives. Patient Prefer Adherence. 2016;10:2021-2029.

30. Jansen F, van Uden-Kraan CF, van Zwieten V, Witte BI, Verdonck-de Leeuw IM. Cancer survivors' perceived need for supportive care and their attitude towards self-management and eHealth. Support Care Cancer. 2015;23(6):1679-1688.

31. Kidd LA. Consequences, control and appraisal: cues and barriers to engaging in self-management among people affected by colorectal cancer - a secondary analysis of qualitative data. Health Expect. 2014; 17(4):565-578.

32. Baudendistel I, Winkler E, Kamradt M, et al. Personal electronic health records: understanding user requirements and needs in chronic cancer care. J Med Internet Res. 2015;17(5):e121.

33. Kunz A, Pohlmann S, Heinze O, et al. Strengthening interprofessional requirements engineering through action sheets: a pilot study. JMIR Hum Factors. 2016;3(2):e25.

34. Kitzinger J. Qualitative research. Introducing focus groups. BMJ. 1995;311(7000):299-302.

35. Baudendistel I, Winkler E, Kamradt M, et al. The patients' active role in managing a personal electronic health record: a qualitative analysis. Support Care Cancer. 2015;23(9):2613-2621.

36. Freund T, Everett C, Griffiths P, Hudon C, Naccarella L, Laurant M. Skill mix, roles and remuneration in the primary care workforce: who are the healthcare professionals in the primary care teams across the world? Int J Nurs Stud. 2015;52(3):727-743. 
37. Malterud K. Qualitative research: standards, challenges, and guidelines. Lancet. 2001;358(9280):483-488.

38. Pope C. Qualitative research in health care: analysing qualitative data. BMJ. 2000;320(7227):114-116.

39. Graneheim UH, Lundman B. Qualitative content analysis in nursing research: concepts, procedures and measures to achieve trustworthiness. Nurse Educ Today. 2004;24(2):105-112.

40. Safford MM, Allison JJ, Kiefe CI. Patient complexity: more than comorbidity the vector model of complexity. J Gen Intern Med. 2007; 22(suppl 3):382-390.

41. Foster C, Roffe L, Scott I, Cotterell P. Self Management of Problems Experienced Following Primary Cancer Treatment: An Exploratory Study. London: Macmillan Cancer Support; 2010.

42. Lorig KR, Holman H. Self-management education: history, definition, outcomes, and mechanisms. Ann Behav Med. 2003;26(1):1-7.

43. Audulv $\AA$. The over time development of chronic illness self-management patterns: a longitudinal qualitative study. BMC Public Health. 2013; 13:452.

44. Schulman-Green D, Jaser S, Martin F, et al. Processes of selfmanagement in chronic illness. J Nurs Scholarsh. 2012;44(2):136-144.

45. Pound P, Gompertz P, Ebrah S. Social and practical strategies described by people living at home with stroke. Health Soc Care Community. 1999;7(2):120-128.

46. Martin F, Kiwanuka T, Kawuma R, Zalwango F, Seeley J. Tasks and strategies of self-management of living with antiretroviral therapy in Uganda. AIDS Patient Care STDS. 2013;27(12):697-706.
47. Bayliss EA, Bosworth HB, Noel PH, Wolff JL, Damush TM, Mciver L. Supporting self-management for patients with complex medical needs: recommendations of a working group. Chronic Illn. 2007;3(2): $167-175$.

48. Bayliss EA, Ellis JL, Steiner JF, Main DS. Initial validation of an instrument to identify barriers to self-management for persons with co-morbidities. Chronic Illn. 2005;1(4):315-320.

49. Bayliss EA, Steiner JF, Fernald DH, Crane LA, Main DS. Descriptions of barriers to self-care by persons with comorbid chronic diseases. Ann Fam Med. 2003;1(1):15-21.

50. Koroukian SM, Xu F, Beaird H, Diaz M, Murray P, Rose JH. Complexity of care needs and unstaged cancer in elders: a population-based study. Cancer Detect Prev. 2007;31(3):199-206.

51. Kamradt M, Baudendistel I, Längst G, et al. Collaboration and communication in colorectal cancer care: a qualitative study of the challenges experienced by patients and health care professionals. Fam Pract. 2015; 32(6):686-693.

52. Baudendistel I, Winkler EC, Kamradt M, et al. Cross-sectoral cancer care: views from patients and health care professionals regarding a personal electronic health record. Eur J Cancer Care (Engl). 2016: doi:10.1111/ecc.12429.

53. Sabaté E. Adherence to Long-Term Therapies: Evidence for Action. Geneva: World Health Organization; 2003. 


\section{Supplementary materials}

Table SI Complexity of individual situations

\begin{tabular}{ll}
\hline Category & Subcategory \\
\hline $\begin{array}{l}\text { Complexity of the } \\
\text { disease }\end{array}$ & $\begin{array}{l}\text { (overall) } \\
\text { Side effects of chemotherapy }\end{array}$ \\
& $\begin{array}{l}\text { Anxiety and uncertainty } \\
\text { Consequences of treatment }\end{array}$ \\
& $\begin{array}{l}\text { Comorbidities and multimorbidity } \\
\text { Complexity of care }\end{array}$ \\
& $\begin{array}{l}\text { Interprofessional and intersectoral } \\
\text { cooperation }\end{array}$
\end{tabular}

Reference

I think cancer patients are very complex patients [...] not only medical but also the psychological aspects [...] the disease is more than just a marathon [FG4-Doc4] I am really satisfied with the therapy except that I have just nausea and constipation and diarrhea and headache, and inflammation in the mouth and so on, and so on, So that comes regularly [FGI-P2]

Patients are often afraid, as it goes on, as is the perspective [FGI0-GP2]

Patients have [.] practical problems: ostomy care [...] impaired wound healing, and so on [FGI0-GP2]

Yes, that's quite a package $[\ldots]$ because I had dealing not only with cancer but as I said to diabetes and then the "vessel cause" [FG2-PI]

I was passed around in the clinic [...] also previously at the preliminary investigation I was at the [...] urologist because [...] the family doctor had a suspicion that I might have kidney stones [...] the urologist sent me to the surgeon and the surgeon sent me to the gynecologist and there I got caught [FG3-P2] Importance of long-term and home A big issue is always the long-term care of the Stoma [FG9-GP4] care So if he has an advanced prognosis patients are cared mainly at home [FG9-GPI] So when people are in working life if have a family [...] that depends very much on in which living situation the people are and how the prognostic situation appears [FG8-T4]

\section{Complexity of (overall)}

treatment data

Increasing amount of data

I have at home a huge paperwork with blood tests and everything [...] and that's just been a long time [FG3-P3]

And then when the patients are in treatment for a long time, then three folders containing medical reports are quickly full [FG8-T2]

Various data sources and numerous data types

Difficult traceability and assignment If the patient has collected results over the past 2 years, it is difficult to decide what is relevant or what not [FG4-Doc3]

All of the data which has emerged, all the medical reports etc. [...] they are all filed away somewhere, but it is always difficulty to reconstruct [...] now and then you lose track [FG2-PI]

Table S2 Strategies of self-management

\begin{tabular}{|c|c|c|}
\hline Category & Subcategory & Reference \\
\hline \multirow[t]{4}{*}{ Proactive demanding } & Support and guidance & $\begin{array}{l}\text { And then I said "please could you tell me where to go now" and then they } \\
\text { told me the next steps and actively cared [FGI-P4] }\end{array}$ \\
\hline & Meaningful talk with & In surgical ward I have actively searched for a conversation and then I \\
\hline & the doctor & complained. Then someone had time to talk with me [FG3-P6] \\
\hline & $\begin{array}{l}\text { Therapy and treatment } \\
\text { options }\end{array}$ & $\begin{array}{l}\text { I took my medical records to the clinic and told "so now we are watching us } \\
\text { this together and discuss something you see" [FG3-P4] }\end{array}$ \\
\hline \multirow[t]{3}{*}{ Proactive behavior } & Organization of care & $\begin{array}{l}\text { I always took care of it. Whether it would have worked even without I do not } \\
\text { know [...] But l've always pushed [FGI-P4] }\end{array}$ \\
\hline & Preparation of visits & $\begin{array}{l}\text { My experience is that it is very helpful if you are well informed goes to the } \\
\text { doctor }[\ldots] \text { all doctors }- \text { I think that's just a certain human weakness - } \\
\text { respond differently to patients who come informed [FG2-PI] }\end{array}$ \\
\hline & Search for information & $\begin{array}{l}\text { I then asked what I can actually eat and then they said "everything". Then I } \\
\text { have to learn [that this is not true]. However, there are excellent booklets on } \\
\text { nutrition for cancer patients [FG2-P2] }\end{array}$ \\
\hline \multirow[t]{3}{*}{$\begin{array}{l}\text { Proactive data } \\
\text { management }\end{array}$} & $\begin{array}{l}\text { Merging of treatment } \\
\text { data }\end{array}$ & $\begin{array}{l}\text { I have all the reports. I am "clamped behind" and have said, hey I still lack and } \\
\text { that is still missing and so on. So it worked well on request [FG3-P2] }\end{array}$ \\
\hline & $\begin{array}{l}\text { Dissemination of } \\
\text { information }\end{array}$ & $\begin{array}{l}\text { And I'll tell it to anyone }[\ldots] \text { they should take and distribute their medical } \\
\text { records }[\ldots] \text { if the patient does not have an extra copy for the doctors, then } \\
\text { he will may be sent back home }[\mathrm{FG} 3-\mathrm{PI}]\end{array}$ \\
\hline & Archiving of documents & I also scan everything again and store it on the computer, as security [FG3-P4] \\
\hline
\end{tabular}




\section{Publish your work in this journal}

Patient Preference and Adherence is an international, peer-reviewed, open access journal that focuses on the growing importance of patient preference and adherence throughout the therapeutic continuum. Patient satisfaction, acceptability, quality of life, compliance, persistence and their role in developing new therapeutic modalities and compounds to optimize

clinical outcomes for existing disease states are major areas of interest for the journal. This journal has been accepted for indexing on PubMed Central. The manuscript management system is completely online and includes a very quick and fair peer-review system, which is all easy to use. Visit http://www. dovepress.com/testimonials.php to read real quotes from published authors.

Submit your manuscript here: http://www.dovepress.com/patient-preference-and-adherence-journal 